\title{
ON GORENSTEIN IDEALS OF CODIMENSION FOUR
}

\author{
W. V. VASCONCELOS ${ }^{1}$ AND R. VILLARREAL
}

\begin{abstract}
We describe two computations showing how the Koszul homology impacts on the structure of Cohen-Macaulay ideals of height 3 and Gorenstein ideals of height 4.
\end{abstract}

1. Introduction. Let $R$ be a Gorenstein local ring and let $I$ be a perfect ideal. One of our aims in this note is to provide a strong link between the Cohen-Macaulayness of two modules associated to $I: H_{1}(I)$, the 1-dimensional homology module of the Koszul complex associated to a system of generators of $I$, and (i) $I / I^{2}$, the conormal module of $I$-in the case of a Gorenstein ideal of height 4, or (ii) $S_{2}(W)$, the symmetric square of the canonical module of $R / I$-in the case of a CohenMacaulay ideal of height 3.

Despite the technical character these results have some palpable applications. Gorenstein ideals of codimension four have not exhibited thus far any structure that approaches the beautiful result of Buchsbaum and Eisenbud [BE] for ideals of height three. In fact, Kustin and Miller (see [KM] and the references there) have uncovered such a diversity of behaviour in codimension four so as to make such an event unlikely. Smaller classes of such ideals do have a regular description, and for our purposes we single out a theorem of Herzog and Miller [HM] on Gorenstein ideals of deviation two. The result here allows, in codimension four, the argument of [HM] to go through in all cases, so that one now has a structure theorem for most Gorenstein ideals with six generators. (One still has to leave out rings in which 2 is not a unit.)

For height 3 ideals the computation is interesting because it shows how often Koszul homology mirrors structure. We have found it particularly useful in some computer analyses being carried out on the structure of ideals with pure resolutions.

The other consequences of our theorems are connected to the blowing-up ring of $I$ and the deformation theory of $I$.

To describe the results we review some definitions. Throughout $R$ will be a local Gorenstein ring, and $I$ is an ideal of height $g$ and finite projective dimension. We denote by $W=\operatorname{Ext}^{g}(R / I, R)$ the canonical module of $S=R / I$.

We shall say that $I$ (or $S$ ) is strongly unobstructed if $I \otimes W$ is Cohen-Macaulay (cf. [He]). Note that if $I$ is a Gorenstein ideal, this last module is just $I / I^{2}$. On the

Received by the editors June 20, 1985 and, in revised form, October 15, 1985.

1980 Mathematics Subject Classification. Primary 13H10; Secondary 13C05, 13C15.

${ }^{1}$ Partially supported by NSF grant DMS-8503004. 
other hand, we denote by $H_{i}(I)$ the homology modules of a Koszul complex built on a set of generators of the ideal. $I$ is said to be strongly Cohen-Macaulay if all the $H_{i}(I)$ are Cohen-Macaulay. (This definition does not depend on the generators chosen.) Further we recall that for a Cohen-Macaulay ideal, being strongly unobstructed is a property of the linkage class of $I$ [BU]. On the other hand, being strongly Cohen-Macaulay-or, more generally, having Cohen-Macaulay Koszul homology up to a given dimension-is preserved along the even linkage class of $I$ $\left[\mathrm{Hu}_{1}\right]$. Finally, the deviation of the ideal $I$ is the deficit $n-g$, where $n$ is the minimum number of generators of $I$ and $g$ is its height. The notation $S_{2}(M)$ will stand for the symmetric square of the $R$-module $M$.

The simplest relationship between $H_{1}(I)$ and $I / I^{2}$ is expressed by the exact sequence

$$
0 \rightarrow \delta(I) \rightarrow H_{1}(I) \rightarrow S^{n} \rightarrow I / I^{2} \rightarrow 0 .
$$

where $\delta(I)=$ kernel of the natural mapping $S_{2}(I) \rightarrow I^{2}$ and may be identified to the deformation module $T_{2}(S, R)$ [SV]. It is tempting to connect $H_{1}(I)$ to $I / I^{2}$ more closely.

(1.1) THEOREM. Let $R$ be a Gorenstein local ring, and let $I$ be a Gorenstein ideal of codimension four. If $H_{1}(I)$ is Cohen-Macaulay then I is strongly unobstructed

This permits stating in greater generality the main theorem of [HM]:

(1.2) TheOREM. Let $R$ be a Gorenstein local ring in which 2 is a unit. Let I be $a$ Gorenstein ideal of codimension four and deviation two. If $I$ is a generic complete intersection, then I is a hypersurface section of a Gorenstein ideal of height three. That is, $I=(J, f)$, where $J$ is the ideal generated by the $4 \times 4$ Pfaffians of an alternating $5 \times 5$ matrix and $f$ is a regular element on $R / J$.

The proof of (1.1) can, in codimension three, be slightly refined.

(1.3) THEOREM. Let $R$ be a Gorenstein local ring, and let I be a Cohen-Macaulay ideal of height three. Then $H_{1}(I)$ is Cohen-Macaulay if and only if $S_{2}(W)$ is Cohen-Macaulay.

We show readily that ideals of type 2 have this property.

2. Proof of (1.1). Our starting point is a minimal projective resolution of $S=R / I$ :

$$
0 \rightarrow R \stackrel{\alpha}{\rightarrow} R^{n} \stackrel{\beta}{\rightarrow} R^{m} \stackrel{\theta}{\rightarrow} R^{n} \rightarrow R \rightarrow S \rightarrow 0 .
$$

We set $\mathbf{a}=\alpha(1)=x_{1} e_{1}+\cdots+x_{n} e_{n}$, where the $e_{i}$ 's form a basis of $R^{n}$, and $\mathbf{x}=\left\{x_{1}, \ldots, x_{n}\right\}$ is a minimal system of generators of $I$. Denote by $\mathbf{K}=\mathbf{K}(\mathbf{x})$ the Koszul complex built on $\mathbf{x} . \mathbf{K}$ is a skew-commutative $R$-algebra, and this structure is passed along to the subalgebra $\mathbf{Z}$ of cycles of $\mathbf{K}$. In particular, there exists a natural mapping

$\left(\#\right.$ \#) $\quad 0 \rightarrow \operatorname{ker}(\lambda) \rightarrow \Lambda^{2} Z_{1} \stackrel{\lambda}{\rightarrow} Z_{2} \rightarrow D=\operatorname{coker}(\lambda) \rightarrow 0$ 
Note that $\lambda$ is an isomorphism whenever $Z_{1}$ is a free module. In fact, we may view $\lambda$ as the natural embedding of $\Lambda^{2} Z_{1}$ into its double dual [HSV]. Before we translate the hypothesis into properties of this complex, let us review the resolution of $\Lambda^{2} Z_{1}$. For that we identify $Z_{1}$ with image $(\theta)$. According to $\left[\mathbf{L e}_{1}, \mathbf{L e}_{2}\right]$, we have an exact sequence

$(\# \#) \quad 0 \rightarrow R \otimes R^{n} \stackrel{\phi}{\rightarrow} R \otimes R^{m} \oplus D^{2}\left(R^{n}\right) \rightarrow R^{m} \otimes R^{n} \rightarrow \Lambda^{2} R^{m} \rightarrow \Lambda^{2} Z_{1} \rightarrow 0$

Here $D^{2}\left(R^{n}\right)$ denotes the divided square of $R^{n}$. We shall make use of the duality between the divided power algebra of a free module $F$ and its symmetric algebra, in particular of a canonical isomorphism $D^{2}(F)^{*} \simeq S_{2}\left(F^{*}\right)$.

$\phi=\left(\phi_{1}, \phi_{2}\right)$ is defined as follows: $\phi_{1}=1 \otimes \beta$ and $\phi_{2}\left(1 \otimes e_{i}\right)=-\mathbf{a} e_{i}$.

Since $\operatorname{pd}_{R}\left(\Lambda^{2} Z_{1}\right)=3$, the associated primes of $\Lambda^{2} Z_{1}$ have height at most three; but as (\#\#) is split exact in codimension less than four, $\operatorname{ker}(\lambda)=0$.

We are now ready to prove (1.1). Set $d=\operatorname{dim}(R)$. The condition $H_{1}(I)=H_{1}(\mathbf{K})$ Cohen-Macaulay means that the module of 1-boundaries of $\mathbf{K}, B_{1}$, has depth $d-3$. Thus, $\operatorname{depth}\left(Z_{2}\right)=d-2$, so that the module $D$, in the complex (\#), has depth $d-4$. Since $D$ vanishes in codimension less than four, it must be a Cohen-Macaulay module of dimension $d-4$. We determine $\operatorname{Ext}^{4}(D, R)$.

The long exact homology sequence obtained by applying $\operatorname{Hom}(-, R)$ to $(\#)$ yields $\operatorname{Ext}^{4}(D, R)=\operatorname{Ext}^{4}\left(\Lambda^{2}, Z_{1}, R\right)$. We claim that this last module is isomorphic to $I / I^{2}$. Note that it is given as $\left(R \otimes R^{n}\right)^{*} / \operatorname{image}\left(\phi_{1}^{*}\right)+\operatorname{image}\left(\phi_{2}^{*}\right)$. But $\left(R \otimes R^{n}\right) /$ image $\left(\phi_{1}^{*}\right)=I$, because the dual of (\#) is again a projective resolution of $R / I$. It is also clear, from the definition of $\phi$ and the identification of $D^{2}\left(R^{n}\right)^{*}$ and $S_{2}\left(R^{n *}\right)$, that image $\left(\phi_{2}^{*}\right)$ maps exactly onto $I^{2}$. As $\operatorname{Ext}^{4}(D, R)$ is Cohen-Macaulay along with $D$ (cf. [HK]), this completes the proof.

Cohen-Macaulay ideals of deviation two are strongly Cohen-Macaulay (cf. [AH]; see also [HVV]), therefore the proof of (1.2) as given in [HM] goes through in its entirety.

REMARK. The condition " $H_{1}(I)$ is Cohen-Macaulay" is not characteristic of Gorenstein ideals of height four. For instance, if $A$ is a generic $3 \times 3$ matrix, and $I$ is the ideal generated by the submaximal minors, then $I$ is Gorenstein of height four but depth $H_{1}(I)=1$ (cf. [Hu 2$]$ ).

3. Proof of (1.3). Given the minimal free resolution of $I$ :

$$
0 \rightarrow R^{p} \rightarrow R^{m} \rightarrow R^{n} \rightarrow I \rightarrow 0 .
$$

we use the method of $\left[\mathbf{L e}_{1}, \mathbf{L e}_{2}\right]$ to construct a minimal resolution of $\Lambda^{2} Z_{1}$ :

$$
0 \rightarrow D^{2}\left(R^{p}\right) \stackrel{\gamma}{\rightarrow} R^{p} \otimes R^{m} \stackrel{\psi}{\rightarrow} \Lambda^{2} R^{m} \rightarrow \Lambda^{2} Z_{1} \rightarrow 0 .
$$

In particular, $\Lambda^{2} Z_{1}$ is a torsion-free module and, as in the proof of (1.1), we obtain the complex

$$
0 \rightarrow \Lambda^{2} Z_{1} \stackrel{\lambda}{\rightarrow} Z_{2} \rightarrow D \rightarrow 0
$$


Again we conclude that $D$ is Cohen-Macaulay if $H_{1}(I)$ is Cohen-Macaulay. The argument in the proof of (1.1) now yields that $\operatorname{Ext}^{3}(D, R) \simeq S_{2}(W)$ is CohenMacaulay as well.

To show the converse, note that the module $D$ may be identified to $\operatorname{Ext}^{2}\left(\operatorname{coker}\left(\psi^{*}\right), R\right)$ (cf. [AB, p. 52]). We must make more explicit the relationship between $D$ and $S_{2}(W)$. Dualize the projective resolution of $\Lambda^{2} Z_{1}$ to obtain the complex

$0 \rightarrow\left(\Lambda^{2} Z_{1}\right)^{*} \rightarrow\left(\Lambda^{2} R^{m}\right)^{*} \stackrel{\psi^{*}}{\rightarrow}\left(R^{p} \otimes R^{m}\right)^{*} \stackrel{\gamma^{*}}{\rightarrow} D^{2}\left(R^{p}\right)^{*}=S_{2}\left(R^{p *}\right) \rightarrow S_{2}(W) \rightarrow 0$.

Set $L=\operatorname{image}\left(\psi^{*}\right), M=\operatorname{image}\left(\gamma^{*}\right), N=\operatorname{ker}\left(\gamma^{*}\right), C=\operatorname{coker}\left(\psi^{*}\right)$. We observe that the support of the module $N / L$ has codimension at least three. Examining the exact sequence

$$
0 \rightarrow N / L \rightarrow\left(R^{p} \otimes R^{n}\right)^{*} / L=C \rightarrow M \rightarrow 0
$$

we obtain that

$$
D=\operatorname{Ext}^{2}(C, R)=\operatorname{Ext}^{2}(M, R)=\operatorname{Ext}^{3}\left(S_{2}(W), R\right) .
$$

Finally, consider the homology sequence obtained by applying $\operatorname{Hom}(-, R)$ to (\# \#):

$$
0 \rightarrow \operatorname{Ext}^{2}\left(Z_{2}, R\right) \rightarrow \operatorname{Ext}^{2}\left(\Lambda^{2} Z_{1}, R\right) \stackrel{\theta}{\rightarrow} \operatorname{Ext}^{3}(D, R) \rightarrow \operatorname{Ext}^{3}\left(Z_{2}, R\right) \rightarrow 0 .
$$

The mid-modules are both isomorphic to $S_{2}(W)$ since, by duality, $S_{2}(W) \simeq$ $\operatorname{Ext}^{3}\left(\operatorname{Ext}^{3}\left(S_{2}(W), R\right), R\right)$. We claim that $\theta$ is an isomorphism by looking at the support of the modules $\operatorname{Ext}^{2}\left(Z_{2}, R\right)$ and $\operatorname{Ext}^{3}\left(Z_{2}, R\right)$. First, if $P$ is an associated prime of $\operatorname{Ext}^{2}\left(Z_{2}, R\right)$-which is embedded in $S_{2}(W)$-it has height three; therefore $\operatorname{Ext}^{2}\left(Z_{2}, R\right)_{p}=0$, since $Z_{2}$ is a second syzygy module. We then have that if $\operatorname{Ext}^{3}\left(Z_{2}, R\right)$ is nonzero, its depth is at least $d-4$-which is impossible since it vanishes in codimension four. We thus obtain that $\operatorname{Ext}^{i}\left(Z_{2}, R\right)=0$ for $i \geqslant 2$, and $\operatorname{depth}\left(Z_{2}\right) \geqslant d-1$, as desired.

RemarK. Having $H_{1}(I)$ Cohen-Macaulay is not enough to ensure that $I$ is strongly Cohen-Macaulay, although this is the case if the deviation of $I$ is at most 4 .

As an application of (1.3) we consider ideals of type 2. As remarked earlier, the 'complementary' case of ideals of deviation two are always strongly Cohen-Macaulay.

(3.1) Proposition. Let $S$ be a Cohen-Macaulay local ring and let $W$ be its canonical module. If the type of $S$ is two, that is, if $W$ is minimally generated by two elements, then $S_{2}(W)$ is Cohen-Macaulay.

Proof. Start with a presentation of $W$

$$
0 \rightarrow L \stackrel{\phi}{\rightarrow} S^{2} \rightarrow W \rightarrow 0 .
$$

Denote by $x, y$ the generators of $W$, images of the basis elements $e_{1}$ and $e_{2}$ of $S^{2}$. This sequence induces a commutative diagram

$$
\begin{gathered}
L \otimes S^{2} \stackrel{\alpha}{\rightarrow} S_{2}\left(S^{2}\right) \\
\beta \searrow \nearrow \gamma \\
S^{2} \otimes S^{2}
\end{gathered}
$$


where $\alpha$ is the canonical mapping such that $\operatorname{coker}(\alpha)=S_{2}(W), \beta=\phi \otimes 1$, and $\gamma$ is the natural surjection. By the snake lemma we have the exact sequence

$$
0 \rightarrow \operatorname{ker}(\beta) \rightarrow \operatorname{ker}(\alpha) \rightarrow \operatorname{ker}(\gamma) \rightarrow \operatorname{coker}(\beta) \rightarrow \operatorname{coker}(\alpha) \rightarrow \operatorname{coker}(\gamma) \rightarrow 0 \text {. }
$$

Observe that $\operatorname{ker}(\beta)=\operatorname{coker}(\gamma)=0, \operatorname{coker}(\beta)=W \oplus W$, and $\operatorname{ker}(\gamma)=S e, e=$ $e_{1} \otimes e_{2}-e_{2} \otimes e_{1}$. We therefore obtain the exact sequence

$$
S e \rightarrow W \oplus W \rightarrow S_{2}(W) \rightarrow 0
$$

where $e$ maps to $(-y, x)$.

Because $W$ is a faithful module, this sequence is also exact at the left. Tensor it with $S /(\mathbf{x})=S^{\prime}$, where $\mathbf{x}$ is a system of parameters of $S$. We obtain the exact sequence

$$
0 \rightarrow \operatorname{Tor}_{1}\left(S^{\prime}, S_{2}(W)\right) \rightarrow S^{\prime} \rightarrow W^{\prime} \oplus W^{\prime} \rightarrow S_{2}\left(W^{\prime}\right) \rightarrow 0 .
$$

We note finally that $W^{\prime}=W \otimes S^{\prime}$ is the canonical module of $S^{\prime}$ and $S^{\prime}$ embeds into $W^{\prime} \oplus W^{\prime}$, as $W^{\prime}$ is faithful. Therefore, $\operatorname{Tor}_{1}\left(S^{\prime}, S_{2}(W)\right)=0$, and $S_{2}(W)$ is Cohen-Macaulay.

(3.2) CoRollary. Let I be a perfect ideal of a ring such as in (1.3). If I has type two, then $H_{1}(I)$ is Cohen-Macaulay.

REMARK. A consequence of (1.3) permits relating the type of $S=R / I$ to its multiplicity. Assume that $I$ is generically a complete intersection and that $H_{1}(I)$ is Cohen-Macaulay. We may identify $W$ to an ideal of $S$; in turn, because $S_{2}(W)$ is Cohen-Macaulay, it may be identified to $W^{2}$ (that is, $W$ is a syzygetic ideal of $S$ ). Set $m=$ multiplicity of $S, p=$ type of $S$. Since the multiplicity is always at least as large as the minimum number of generators of Cohen-Macaulay ideals, $m \geqslant\left[{ }_{2}^{p+1}\right]$. Let us illustrate this with the case of a pure resolution. Assume $R=k\left[x_{1}, \ldots, x_{n}\right]$ and that $I$ is an ideal generated by forms of degree $d$, whose higher syzygies are also generated by elements of the same degree:

$$
0 \rightarrow R^{p}(-d-a-b) \rightarrow R^{q}(-d-a) \rightarrow R^{r}(-d) \rightarrow R \rightarrow S \rightarrow 0 .
$$

According to the formulae of [HKu], the type of $S$ is given by

$$
p=d(d+a) / b(a+b),
$$

while by [HuM] the multiplicity of $S$ is $m=d(d+a)(d+a+b) / 6$. Therefore we conclude that if $H_{1}(I)$ is Cohen-Macaulay, then

$$
3(d(d+a)+b(b+a)) \leqslant(d+a+b)(b(b+a))^{2} .
$$

We expect ideals of this kind to be very scarce. A computer analysis has shown that if further $I$ has deviation three, or deviation four and it is not Gorenstein, then $H_{1}(I)$ cannot be Cohen-Macaulay.

\section{REFERENCES}

[AB] M. Auslander and M. Bridger, Stable module theory, Mem. Amer. Math. Soc. no. 94 (1969).

[AH] L. Avramov and J. Herzog, The Koszul algebra of a codimension two embedding, Math. Z. 175 (1980), 249-260.

[BE] D. Buchsbaum and D. Eisenbud, Algebra structures for finite free resolutions and some structure theorems for ideals of codimension 3, Amer. J. Math. 99 (1977), 447-485. 
[BU] R. O. Buchweitz and B. Ulrich, Homological properties which are invariant under linkage, preprint, 1983.

[He] J. Herzog, Deformation von Cohen-Macaulay algebren, J. Reine Angew. Math. 318 (1980), 83-105.

[HKu] J. Herzog and M. Kühl, On the Betti numbers of finite pure and linear resolutions, Comm. Algebra 12 (1984), 1627-1646.

[HK] J. Herzog and E. Kunz, Der kanonische Modul eines Cohen-Macaulay rings, Lecture Notes in Math., vol. 283, Springer-Verlag, Berlin and New York, 1971.

[HM] J. Herzog and M. Miller, Gorenstein ideals of deviation two, Comm. Algebra 13 (1985), 1977-1990.

[HVV] J. Herzog, W. V. Vasconcelos and R. Villarreal, Ideals with sliding depth, Nagoya Math. J. 99 (1985), 159-172.

[HSV] J. Herzog, A. Simis and W. V. Vasconcelos, On the arithmetic of algebras of linear type, Trans. Amer. Math. Soc. 283 (1984), 661-683.

$\left[\mathrm{Hu}_{1}\right]$ C. Huneke, Linkage and the Koszul homology of ideals, Amer. J. Math. 104 (1982), 1043-1062.

$\left[\mathrm{Hu}_{2}\right] \ldots$ Determinantal ideals of linear type, preprint, 1985.

[HuM] C. Huneke and M. Miller, A note on the multiplicity of Cohen-Macaulay algebras with pure resolutions, Canad. J. Math. (to appear).

[KM] A. Kustin and M. Miller, Deformation and linkage of Gorenstein algebras, Trans. Amer. Math. Soc. 284 (1984), 501-534.

[Le $\left.{ }_{1}\right]$ K. Lebelt, Zur homologischen dimension äußerer Potenzen von Moduln, Arch. Math. 26 (1975), $595-601$.

[Le $\left.{ }_{2}\right] \ldots$, Freie Auflösungen äußerer Potenzen, Manuscripta Math. 21 (1977), 341-355.

[SV] A. Simis and W. V. Vasconcelos, The syzygies of the conormal module, Amer. J. Math. 103 (1981), 203-224.

Department of Mathematics, Rutgers University, New Brunswick, New Jersey 08903 